\title{
Bacterial Circadian Programs
}

\author{
C.H. JoHnson \\ Department of Biological Sciences, Vanderbilt University, \\ Nashville, Tennessee 37235
}

\begin{abstract}
Twenty years ago, it was widely believed that prokaryotes were too "simple" to have evolved circadian programs. Since that time, however, the cyanobacterial circadian system has progressed from a curiosity to a major model system for analyzing clock phenomena. In addition to globally regulating gene expression, cyanobacteria are one of the only systems in which the adaptive fitness of a circadian system has been rigorously evaluated. Moreover, cyanobacteria are the only clock system in which all essential proteins of the core oscillator have been crystallized and structurally determined, namely, the KaiA, KaiB, and KaiC proteins. A biochemical oscillator can be reconstituted in vitro with these three purified Kai proteins and displays the key properties of temperature-compensated rhythmicity. This result spectacularly demonstrates that a strictly posttranslational clock is sufficient to elaborate circadian phenomena and that a transcription-translation feedback loop is not obligatory. The conjunction of structural information on essential clock proteins with a defined system that reconstitutes circadian oscillations in vitro leads to a turning point whereby biophysical and biochemical approaches bring analyses of circadian clockwork to an unprecedented level of molecular detail.
\end{abstract}

\section{INTRODUCTION}

At the original Cold Spring Harbor Symposium on Biological Clocks (47 years ago), there was already an appreciation that microbial organisms were capable of circadian rhythmicity. That volume featured papers on circadian rhythms in the microbes Euglena (Bruce 1961), Gonyaulax (Hastings 1961; Sweeney 1961; Sweeney and Hastings 1961), and Paramecium (Ehret 1961). Several of those papers have been influential for many years. For example, the paper by J.W. Hastings on biochemical aspects has been widely cited as evidence for the relative insensitivity of circadian oscillators to drugs/chemicals (Hastings 1961). The paper by B.M. Sweeney on single Gonyaulax cells provided suggestive evidence that circadian rhythms might be a cellular phenomenon and not an emergent property of populations of cells (Sweeney 1961). And the paper by Sweeney and Hastings on temperature compensation is still cited regularly for its suggestion that temperature compensation might be mechanistically accomplished by coupled biochemical reactions (Sweeney and Hastings 1961). During the past 47 years, salient discoveries in circadian rhythmicity have used microbial organisms (Edmunds 1988; Johnson and Kondo 2001). The two eukaryotic microbes that are most commonly studied today for circadian investigations are the fungus Neurospora crassa and the green alga Chlamydomonas reinhardtii, whose circadian properties were first reported in 1959 for Neurospora (Pittendrigh et al. 1959) and in 1970 for Chlamydomonas (Bruce 1970). Undoubtedly, the reason that those two microbes have been selected for continued study is that both classical and molecular genetic approaches are possible.

Nevertheless, with the increasing ability in mammalian systems to apply techniques that were heretofore only possible in microbes, coupled with the increasing pressure from funding agencies to do "translational research" rather than basic research, the interest in nonmammalian models for the study of circadian rhythms has waned somewhat. This attitude might be particularly applied to the case of bacterial model systems - the subject of this paper-because the key clock genes that are involved in prokaryotic circadian rhythms (at least, in cyanobacteria; Ishiura et al. 1998) have no homologs in the genomes of mammals, insects, or fungi, nor do the clock genes in eukaryotes have obvious homologs in bacteria. Despite that perception, however, the bacterial circadian system has in the interval of 15 years blossomed from a curiosity to one of the most important model systems - one that has enabled insights and approaches that were technically impossible elsewhere. The goal of this chapter is to briefly describe the insights resulting from the study of bacterial (especially cyanobacterial) clocks, to demonstrate what those studies have uniquely told us, and to speculate upon the future of bacterial clock studies and what they might tell us about eukaryotic clocks.

\section{THE DISCOVERY OF CIRCADIAN CLOCKS IN BACTERIA}

Before 1986, it was generally thought that circadian rhythms were exclusively a eukaryotic phenomenon (Ehret 1961; Johnson et al. 1996). A few reports that suggested that bacteria might have circadian clocks were not persuasive because the described rhythms were noisy and temperature compensation had not been demonstrated (Halberg and Conner 1961; Sturtevant 1973). That prokaryotic cells were too "simple" to express circadian behavior became a dogma (Johnson et al. 1996), despite the fact that there were few publications that directly tested the proposition (one exception was Taylor 1979). Some models relied on this dogma as an a priori assumption upon which eukaryotic intracellular organelles were 
conceived to be a necessary prerequisite for a circadian mechanism (Ehret and Trucco 1967; Schweiger and Schweiger 1977; Kippert 1986).

During 1985-1986, three papers were published that began to depose the "no clocks in proks" dogma (Stal and Krumbein 1985; Grobbelaar et al. 1986; Mitsui et al. 1986). These various groups discovered that cyanobacteria which fix nitrogen (Oscillatoria sp. strain 23; Synechococcus spp. Miami BG 43511 and 43522; Synechococcus sp. RF-1) display daily rhythms of nitrogen fixation in LD (light/dark) and in LL (light/light). In particular, the group of Huang and coworkers was apparently the first to clearly recognize that cyanobacteria were exhibiting circadian rhythms and, in a series of publications beginning in 1986, demonstrated the salient characteristics of circadian rhythms in the unicellular freshwater cyanobacterium, Synechococcus sp. RF-1 (Grobbelaar et al. 1986; Huang and Grobbelaar 1995).

A crucial characteristic that needed to be demonstrated for cyanobacteria before they could be accepted into the circadian fold was that of temperature compensation. This criterion was first satisfied by studies of two species of Synechococcus: the marine Synechococcus WH7803 and the freshwater Synechococcus RF-1. Sweeney and Borgese (1989) showed that WH7803 displays temperature-compensated rhythms of cell division, whereas Chen et al. (1991) found temperature-compensated rhythms of amino acid uptake in the freshwater cyanobacterium isolated from rice fields, Synechococcus RF-1. These findings along with those of others unseated the eukaryotic-centric dogma of chronobiology (Johnson et al. 1996). Despite the fact that this dogma is a relic of the past, however, cyanobacteria remain the only prokaryotic system for which circadian organization is proven at this time (see below).

Our laboratory and those of our collaborators-Drs. Takao Kondo, Susan Golden, and Masahiro Ishiuraextended the studies on circadian programming to nonnitrogen-fixing cyanobacteria that were genetically malleable (Johnson et al. 1996). Our first studies used a strain of Synechococcus elongatus PCC 7942 transformed with a luminescence reporter construct, which is the fusion of the $p s b A I$ promoter with a bacterial luciferase cassette $\left(\mathrm{P}_{p s b A i}:\right.$ :luxAB; Kondo et al. 1993). This strain of cyanobacteria is notable because of the ease with which exogenous DNA can be transformed and homologously recombined into the chromosome (Andersson et al. 2000). Because the circadian clock turns $\mathrm{P}_{p s b A I}$ on and off rhythmically, this first reporter strain of $S$. elongatus glowed rhythmically. This choice of strain and promoter was fortuituous. Subsequent experiments using other strains/species of cyanobacteria have found rhythms (Aoki et al. 1997), but the reporters in those strains are not bright. And even in S. elongatus, other promoters do not show such a robust rhythm of luminescence (Liu et al. 1995). The combination of the $\mathrm{P}_{p s b A I}::$ luxAB reporter and the $S$. elongatus strain remains one of the most robustly rhythmic combinations in cyanobacteria, even after 15 years of intensive research. Dr. Kondo was able to exploit the bright and robust luminescence rhythm by designing clever apparatuses to monitor the rhythms. For liquid cultures, Dr. Kondo enlisted and modified the automated photomultiplier apparatus that was originally designed for endogenously bioluminescent algae (Kondo et al. 1993). For monitoring colonies, Drs. Kondo and Ishiura discovered that the rhythms of single colonies could be tracked with a charge-coupled device (CCD) camera (Kondo and Ishiura 1994). Dr. Kondo used those observations to design an innovative turntable/CCD camera apparatus for mutant screening of single colonies, enabling the simultaneous screening of up to 12,000 colonies in a single experiment (Kondo et al. 1994).

The coincidence of good fortune, clever ideas, and hard work has transformed the $S$. elongatus system into one of the best-characterized circadian clock systems, even though it is a relative newcomer to molecular clock analyses; only 20 years ago, no one believed that prokaryotes were capable of circadian rhythmicity (Johnson et al. 1996). Therefore, the S. elongatus system has rapidly caught up with and in several areas has surpassed the eukaryotic systems, as described below.

\section{CIRCADIAN ORCHESTRATION OF GLOBAL GENE EXPRESSION}

With bacteria finally accepted as "members of the circadian club," an early question of interest was how pervasive was clock control of gene expression in the $S$. elongatus system. Was psbAI an isolated example of a gene that was regulated by the circadian mechanism or were more genes under its control in S. elongatus? In eukaryotes, the number of genes regulated by the circadian clock ranges between $5 \%$ and $15 \%$, but eukaryotic genetic organization and regulation are very different from that of prokaryotes. For example, with the exception of a few genes on plasmids, all the genes of S. elongatus are arrayed upon a single circular chromosome. Might they be regulated coordinately?

This question was answered by a sensitive and comprehensive promoter-trap experiment (Liu et al. 1995). A promoterless luciferase gene set was randomly inserted throughout the genome; whenever the luciferase gene set inserted into a genomic locus that was correctly positioned and oriented to a promoter, luciferase was expressed and the colonies glowed. The luminescent patterns of more than 800 independent colonies were analyzed. We were astonished to observe that all of the glowing colonies displayed circadian rhythms with the same period (Liu et al. 1995). The pattern of the rhythmic expression differed among the promoters, both in terms of phasing and in waveform (Liu et al. 1996). The promoter activity for a ribosomal RNA gene $(r r n A)$ was likewise rhythmic (Liu et al. 1995), as well as heterologous promoters, such as an Escherichia coli promoter $\left(\mathrm{P}_{\text {conII }}\right)$ that is transcribed rhythmically when inserted into the cyanobacterial chromosome (Katayama et al. 1999). Apparently, the cyanobacterial clock globally controls gene expression, in this case, the activity of all promoters. This global expression is regulated by one of the key cyanobacterial clock proteins (KaiC; see below) (Nakahira et al. 2004). It is possible that these patterns are mediated by daily changes in the topology of the single chromosome which modulate the activity of all promoters 


\section{BACTERIAL CIRCADIAN PROGRAMS}

in the chromosome (Mori and Johnson 2001a; Smith and Williams 2006; Woelfle and Johnson 2006). If so, the cyanobacterial chromosome might be envisioned as an oscillating nucleoid, or "oscilloid" that regulates all promoters by torsion-sensitive transcription (Johnson 2004; Woelfle and Johnson 2006; M.A. Woelfle et al. 2007).

\section{TWO TIMING CIRCUITS IN THE SAME CELL? CELL DIVISION VERSUS CIRCADIAN OSCILLATORS}

There is a long history of study of the interplay between the cell division cycle and the circadian system in microbes (Edmunds 1988; Mori and Johnson 2000); this is a topic that vertebrate biologists appear to have recently "rediscovered" (Hunt and Sassone-Corsi 2007). In microbes, the bottom line is that the circadian clockwork is independent of cell division timing (otherwise, how could the circadian clock keep accurate track of environmental time?) but that the circadian clock gates the timing of cell division by having a circadian checkpoint in the cell division cycle. This circadian checkpoint assures that cell division will occur at an optimal environmental phase.

The independence of the circadian system from cell division cycling is clear in $S$. elongatus. These bacteria express robust circadian rhythms of gene expression when dividing with doubling times much faster than once per 24 hours (at least down to doubling times of 6-12 hours; Mori et al. 1996: Kondo et al. 1997). They also express excellent rhythms when a key cell division cycle protein, FtsZ, is overexpressed: Division is inhibited but growth continues to produce long "spaghetti-like" cells (Fig. 1) (Mori and Johnson 2001b). Therefore, the circadian clock appears to ignore the status of the cell division cycle in cyanobacteria. However, the timing of cell division is not independent of the circadian clock; even when the cells are dividing rapidly, the circadian clock rhythmically slows the rate of cell division every night. Even in constant light, the rate of cell division is slowed in the early subjective night as though there is a circadian checkpoint that forbids division in the early night (Mori et al. 1996). Apparently, the circadian clockwork is wellbuffered and stable against significant changes of the intracellular milieu so that it can accomplish its raison d'etre-keeping track of environmental phase and regulating cellular events to occur at the optimal time. Consequently, the circadian clockwork in S. elongatus gates cell division and gene expression, but its timing circuit is independent of the cell division cycle.

\section{ADAPTIVE SIGNIFICANCE OF CIRCADIAN TIMING IN CYANOBACTERIA}

The first studies of cyanobacterial clocks used diazotrophic strains that fix nitrogen at night and photosynthesize in the day (Grobbelaar et al. 1986; Mitsui et al. 1986). Because photosynthesis generates oxygen that inhibits the nitrogen-fixing enzyme nitrogenase, this day/night "temporal separation" seemed to be a clear case of the adaptiveness of circadian programming. This hypothesis would predict that cyanobacterial growth in

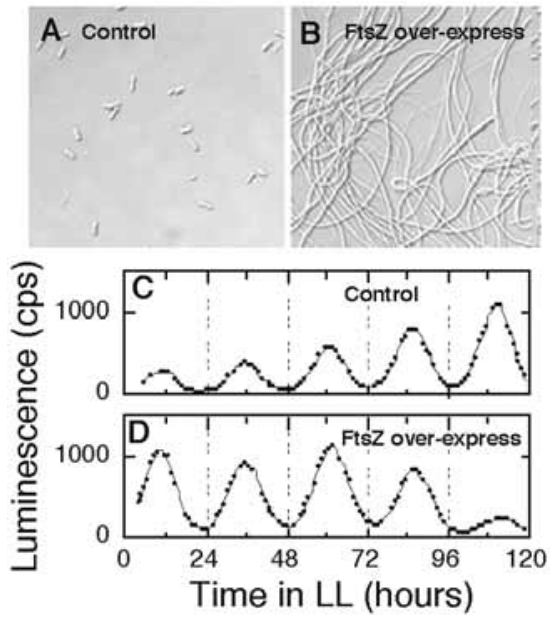

Figure 1. Circadian rhythms of gene expression continue in dividing versus nondividing cells of $S$. elongatus. Cell division of growing cyanobacteria is stopped by overexpression of the cell septum protein FtsZ. $(A, B)$ Morphology of control cells $(A)$ and cells in which FtsZ is overexpressed $(B) .(C, D)$ Luminescence rhythms in control cells $(C)$ and cells in which division has been halted by FtsZ overexpression (D). Luminescence rhythms are monitored from reporters of luciferase-promoter fusions (Kondo et al. 1993). The reporter in $C$ and $D$ is the kaiBC promoter driving bacterial luciferase expression. (Modified, with permission, from Mori and Johnson 2001b [C American Society for Microbiology].)

constant light would be slower than in a light/dark cycle because nitrogen fixation would be inhibited under these conditions, and therefore the growing cells might rapidly become starved for metabolically available nitrogen. The problem is that cyanobacteria grow perfectly well in constant light; in fact, they grow faster in constant light than in light/dark cycles, presumably because of the extra energy they derive from the additional photosynthesis. This result is inconsistent with the "temporal separation" hypothesis. This is an example of "adaptive storytelling" (Johnson 2005). A more rigorous test was needed.

We therefore tested the adaptive significance of circadian programs in the S. elongatus by competing different strains against each other in different laboratory environments (Ouyang et al. 1998; Woelfle et al. 2004). For asexual microbes such as $S$. elongatus, differential growth of one strain under competition with other strains is a good measure of reproductive fitness. In pure culture, the strains grew at about the same rate in constant light and in light/dark cycles, so there did not appear to be a significant advantage or disadvantage in having different circadian periods when the strains were grown individually. The fitness test was to mix different strains together and to grow them in competition to determine if the composition of the population changes as a function of time. The laboratory environments were different kinds of light/dark (LD) cycles or constant light. The cultures were diluted at intervals to allow growth to continue.

In one series of experiments, wild-type cells were competed against an apparently arhythmic strain (CLAb). As shown in Figure $2 \mathrm{~B}$, the arhythmic strain was rapidly defeated by wild type in LD 12:12, but under competition 


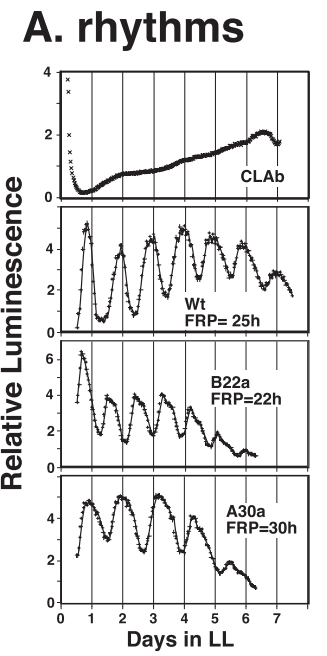

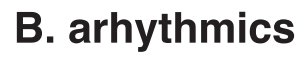

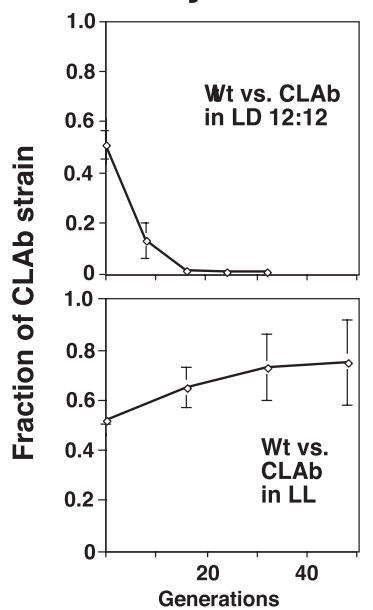

C. FRP mutants

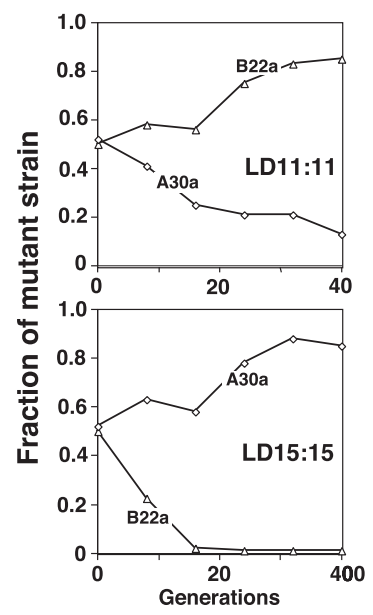

Figure 2. Competition of clock-modified strains with wild type in mixed cultures of $S$. elongatus. (A) Circadian phenotypes of luminescence emission from wild type, the clock-disrupted strain CLAb, and period mutants (mutation in kaiB [B22a, period 22 hours] and kaiA [A30a, period 30 hours]). (B) Competition between wild type and CLAb in LD 12:12 (upper) and LL (lower) plotted as the fraction of mutant in the mixed culture versus the estimated number of generations (mean \pm S.D.). (C) Kinetics of competition between wild type and the period mutant strains in mixed cultures exposed to LD 11:11 (upper) or LD 15:15 (lower). Data are plotted as the fraction of the mutant strain in the mixed culture versus the estimated number of generations. (FRP) Free-running period; (LD) light/dark cycle; (LL) constant light. (Modified, with permission, from Woelfle et al. 2004 [@ Elsevier].)

in constant light, the arhythmic strain grew slightly better than wild type (Woelfle et al. 2004). Therefore, the clock system does not appear to confer an intrinsic value for cyanobacteria under constant conditions. In another series of experiments, period mutants were used to answer the question: Does having a period that is similar to the period of the environmental cycle enhance fitness? The circadian phenotypes of the strains shown in Figure 2A had freerunning periods of about 22 hours (B22a) and 30 hours (A30a) (wild type has a period of about 25 hours under these conditions). When each of the strains was mixed with another strain and grown together in competition, a pattern emerged that depended on the frequency of the light/dark cycle and the circadian period. When grown on a 22-hour cycle (LD 11:11), the 22-hour-period mutant could overtake wild type in the mixed cultures (Fig. 2C). On the other hand, in a 30-hour cycle (LD 15:15), the 30hour-period mutant could defeat wild type (Fig. 2C). On a "normal" 24-hour cycle (LD 12:12), the wild-type strain could outcompete either mutant (Ouyang et al. 1998; Woelfle et al. 2004). Note that over many cycles, each of these light/dark conditions have equal amounts of light and dark (which is important because photosynthetic cyanobacteria derive their energy from light); it is only the frequency of light versus dark that differs among the light/dark cycles.

These data clearly show that the strain whose period most closely matched that of the light/dark cycle eliminated the competitor. Because the mutant strains could defeat the wild-type strain in light/dark cycles in which the periods are similar to their endogenous periods, the differential effects that were observed are likely to result from the differences in the circadian clock. Our results show that an intact clock system whose free-running period is consonant with the environment significantly enhances fit- ness of cyanobacteria in rhythmic environments; however, this same clock system provides no adaptive advantage in constant environments and may even be slightly detrimental to this organism. We still do not know the mechanistic basis for these competitive effects in cyanobacteria, especially because pure cultures of each strain seem to have no decrement in growth. Does this mean that the basis of the competition results is in the interaction between strains in mixed cultures? Despite the fact that we do not know the mechanism by which one strain outcompetes another, however, our competition approach inspired similar studies with the plant Arabidopsis, where the competitive advantage under conditions of clock/environment consonance was attributed to differences in photosynthetic efficiency (Dodd et al. 2005).

\section{STRUCTURAL BIOLOGY OF CIRCADIAN CLOCK PROTEINS}

A mutation/complementation analysis identified the three key clock genes in S. elongatus: kaiA, kaiB, and kaiC, which are clustered together on the chromosome (Ishiura et al. 1998). As described below, the proteins encoded by these genes are necessary and sufficient for circadian rhythmicity (at least, for in vitro rhythmicity). The years 2004-2005 marked a dramatic turning point in the circadian clock field, with the nearly simultaneous reporting of the three-dimensinal structures of the KaiA, KaiB, and KaiC proteins - the first clock proteins from any system to have their full-length structures defined. Cyanobacteria remain the only clock system in which all essential proteins of the core oscillator have been crystallized and structurally determined. This event is important because structural information enables analyses at a truly molecular level; now, we can interpret the effect of mutations on the 
structure and function of the clock proteins. We can also make precise predictions about the ways in which clock proteins interact and influence each other's activity.

The structure of cyanobacterial KaiA was studied by several groups, and solved by solution nuclear magnetic resonance (NMR) (Vakonakis et al. 2004) and X-ray crystallography (Garces et al. 2004; Ye et al. 2004). The 2.0- $\AA$ crystal structure of KaiA from $S$. elongatus reveals that the protein is composed of two independently folded domains connected by a linker (Fig. 3) (Ye et al. 2004). The aminoterminal pseudoreceiver domain has a fold similar to that of bacterial response regulators (despite a lack of sequence similarity), whereas the carboxyl-terminal four-helix bundle domain is novel and forms the interface of the twofoldrelated homodimer. The crystal structure of cyanobacterial KaiB revealed an $\alpha-\beta$ meander motif (Garces et al. 2004; Hitomi et al. 2005). The fold shows close resemblance to the $\alpha-\beta$ motif of thioredoxin. Although the folds of KaiA versus KaiB are clearly different, their size and some surface features of the physiologically relevant dimers are very similar. Notably, the functionally important residues Arg-69 of KaiA and Arg-23 of KaiB align well in space. The apparent structural similarities suggest that KaiA and KaiB may compete for a potential common binding site on KaiC (Garces et al. 2004; Hitomi et al. 2005), and these similarities may explain KaiB's ability to antagonize the effects of KaiA upon KaiC phosphorylation.

My laboratory collaborated with our structural colleagues Drs. Martin Egli, Rekha Pattanayek, and Jimin Wang to determine the crystal structure of the core protein of the circadian clock system from $S$. elongatus, the KaiC homohexamer, at $2.8-\AA$ resolution (Pattanayek et al. 2004). The structure resembles a double doughnut with a central pore that is partially sealed at one end (Fig. 3). This crystal structure revealed ATP-binding sites, intersubunit organization, a scaffold for Kai protein complex formation, the location of critical KaiC mutations, and evolutionary relationships to other proteins such as DnaB and RecA. The KaiCI and KaiCII domains of each subunit adopt similar conformations, but their ATP-binding pockets exhibit significant differences that are likely of functional importance. Previously identified mutations that affect rhythmicity and length of period map to the subunit interface and KaiA-binding regions. The most important binding site of KaiA to KaiC appears to be on the surface of the CII terminus and to 22-amino-acid "tentacles" that extend from CII (Vakonakis and LiWang 2004; Pattanayek et al. 2006). In addition to facilitating our understanding of KaiA-KaiC interaction, the KaiC structure also sheds light on the mechanism of rhythmic phosphorylation of KaiC by identifying T432, S431, and T426 in KaiCII as sites at which KaiC is phosphorylated (Xu et al. 2004); an independent mass spectrometry approach also identified T432 and S431 but not T426 (Nishiwaki et al. 2004). The important role of these residues is confirmed by the loss of rhythmicity in T432A, S431A, and T426A single mutants (Nishiwaki et al. 2004; Xu et al. 2004).

\section{THE CYANOBACTERIAL CLOCKWORK: A TRANSCRIPTIONAL-TRANSLATIONAL FEEDBACK LOOP? OR NOT?}

At the time that the central cyanobacterial clock genes kaiABC were reported (Ishiura et al. 1998), it appeared that the cyanobacterial clockwork was a transcriptional-translational feedback loop (TTFL) oscillator as had been pro-
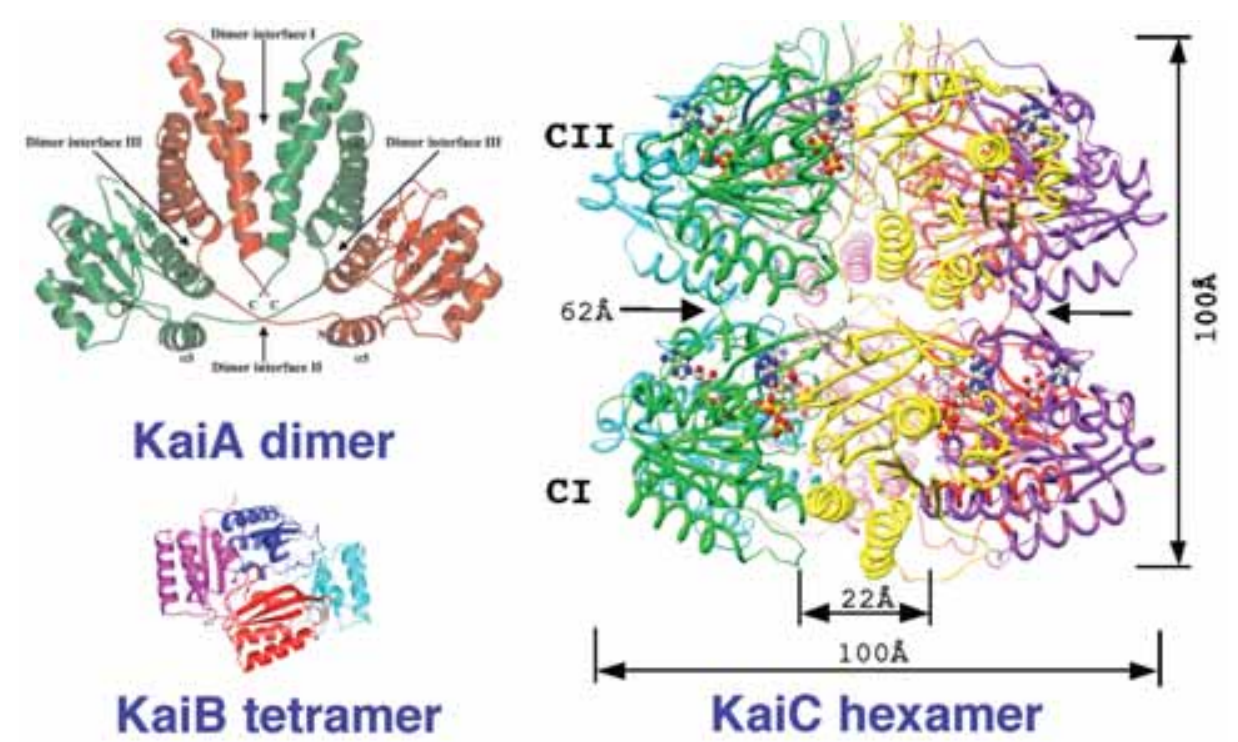

Figure 3. Ribbon representations of the three essential cyanobacterial clock proteins determined from crystal structures. (Upper left) Dimer of KaiA from S. elongatus. Different colors denote the two different KaiA molecules in the dimer. (Lower left) Tetramer of the KaiB from Synechocystis sp. PCC6803; different colors indicate the four KaiB molecules in the tetramer. (Right) Hexamer of KaiC from $S$. elongatus viewed from the side. Individual subunits are colored differently; the slimmer waist region of the particle demarcates the CI and CII domains. ATP molecules are drawn in a ball-and-stick mode with atoms colored gray, red, blue, and yellow for carbon, oxygen, nitrogen, and phosphorus, respectively. (Upper left, modified, with permission, from Ye et al. 2004 [C American Society for Biochemistry and Molecular Biology]; lower left, modified, with permission, from Hitomi et al. 2005 [C American Society for Biochemistry and Molecular Biology]; right, modified, with permission, from Pattanayek et al. 2004 [C Elsevier].) 
posed for the circadian mechanism in various eukaryotic organisms (Dunlap 1999). This inference was based on the fact that the same kind of evidence used to support a TTFL oscillator in eukaryotes also held true for the cyanobacterial clock, namely: (1) rhythms of abundance for mRNAs and proteins encoded by "clock genes," (2) negative feedback of clock proteins on their gene's transcription, and (3) phase setting by overexpression of clock genes, and others. However, recent experiments have led to a major reevaluation of the roles of the TTFL versus a specifically posttranslational oscillator in the cyanobacterial system. The first indication that the cyanobacterial TTFL model was flawed was the demonstration that the promoters driving kaiABC gene expression could be replaced with nonspecific heterologous promoters without disturbing the circadian rhythm (Xu et al. 2003; Nakahira et al. 2004). If the TTFL model were correct in its original formulation, the Kai proteins would be expected to feedback-inhibit their own transcription through direct or indirect interaction with their specific promoters. By that line of reasoning, if the kaiABC promoters were replaced with nonspecific heterologous promoters, the TTFL model would predict that the central feedback loop would be interrupted. But this did not happen in the cyanobacterial case (Xu et al. 2003; Nakahira et al. 2004).

Studies of cyanobacterial cells in dark/dark more directly attacked the TTFL model. Because S. elongatus is an obligate photoautotroph, transcription and translation are shut down in darkness, but Tomita et al. (2005) discovered that the rhythm of KaiC phosphorylation continues robustly in dark/dark. They also discovered that pharmacological inhibition of transcription or translation similarly had little effect upon the circadian rhythm of KaiC phosphorylation. Therefore, neither transcription nor translation was needed to accomplish the circadian rhythm of posttranslational modification of KaiC. The coup de grâce for the usual formulation of the TTFL model came with the stunning demonstration that the three Kai proteins could be combined in a test tube and the circadian rhythm of KaiC phosphorylation merrily churned along! (Nakajima et al. 2005) This in vitro rhythm was even temperature-compensated, so the temperature-compensation mechanism was also encoded in the characteristics of the three Kai proteins and the nature of their interaction. Clearly, transcriptional and translational feedback is not necessary to build a circadian clockwork. Does this mean that transcriptional and translational feedback has no role in the larger circadian system in cyanobacteria? Not necessarily. It is still possible - even likely - that the rhythms of Kai protein abundance can feed into the posttranslational oscillator to promote robustness, and the posttranslational oscillator regulates the timing of transcription-translation to occur in the optimal phase so as to promote that robustness. If so, it may be that the posttranslational oscillator is embedded in a TTFL; the posttranslational oscillator is the process that most directly determines the dynamics of the circadian system, but the TTFL can affect the system by providing a secondary feedback loop. The elucidation of the relative contributions of the posttranslational KaiABC oscillator versus the TTFL circuit is therefore of key importance for future studies.

\section{VISUALIZING THE TICKING OF THE CYANOBACTERIAL IN VITRO CLOCKWORK}

The availability of an in vitro system for analyzing the molecular nature of a circadian clockwork permits biophysical, biochemical, and structural analyses that were previously impossible. Given that the rubicon of threedimensional structural knowledge had been crossed for the Kai proteins, we embraced these techniques and collaborated with Drs. Phoebe Stewart and Dewight Williams to use electron microscopy (EM) to analyze and quantify the time-dependent interaction among the three proteins (KaiA, KaiB, and KaiC) to elucidate the timing of the formation of complexes among the Kai proteins (Mori et al. 2007). We could visualize in the EM that KaiC existed in any of all possible combinations at the different phases of the in vitro oscillation, namely, free KaiC hexamers, $\mathrm{KaiA} \bullet \mathrm{KaiC}$ complexes, KaiB•KaiC complexes, and KaiA• $\mathrm{KaiB} \cdot \mathrm{KaiC}$ complexes, but the proportions of these complexes vary in a phase-dependent manner: Free KaiC hexamers predominate at all phases, about $10 \%$ of KaiC hexamers appear as $\mathrm{KaiA} \cdot \mathrm{KaiC}$ complexes at all phases, and $\mathrm{KaiB} \cdot \mathrm{KaiC}$ and $\mathrm{KaiA} \cdot \mathrm{KaiB} \cdot \mathrm{KaiC}$ complexes are clearly rhythmic with a peak in the KaiC dephosphorylation phase. These results were confirmed by two-dimensional native gel electrophoresis (Mori et al. 2007) and are consistent with the results obtained by the Kondo lab using other techniques (Kageyama et al. 2006). The Kondo lab had reported that KaiC hexamers appeared to be capable of exchanging monomers among the hexamers; therefore, we used the biophysical technique of fluorescence resonance energy transfer (FRET) to confirm that monomer exchange among KaiC hexamers occurs. Finally, we applied the first perturbation analyses of the in vitro oscillator by using temperature pulses to reset the phase of the KaiABC oscillator, thereby testing the resetting characteristics of this unique circadian oscillator (Mori et al. 2007). These structural, biochemical, and biophysical approaches have provided insights to a circadian clockwork at a level of molecular detail that was heretofore unthinkable (Kageyama et al. 2006, Mori et al. 2007).

\section{MODELING THE IN VITRO OSCILLATOR}

I have always been an advocate of the potential benefits of modeling toward opening our eyes to features of circadian rhythmicity that are not otherwise obvious. For example, I firmly believe that limit-cycle modeling of phase resetting has been enlightening (Johnson et al. 2003). Nonetheless, my laboratory has not contributed any mathematical model of circadian mechanisms because I felt that our knowledge of the "gears and cogs" of circadian oscillators was too rudimentary to provide the basis for a useful model of the circadian mechanism. Until now, that is! When the Kondo lab provided a circadian clockwork that depended only on three purified proteins in a test tube (Nakajima et al. 2005), I decided that the time had come for a predictive mathematical model that would be testable by biochemical, biophysical, and structural experiments. Therefore, the structural and biophysical data from our laboratory (Mori et al. 2007) and the biochemical data from the Kondo lab (Kageyama et 
al. 2006) were used by our physicist collaborator Dr. Mark Byrne to derive a dynamic model for the in vitro oscillator that accurately reproduced the rhythms of KaiABC complexes and of KaiC phosphorylation.

Figure 4 depicts this model, which stochastically simulates the kinetics of KaiC hexamers and the degree of phosphorylation of each monomer in every hexamer. Starting from a hypophosphorylated state of KaiC (state $\alpha$ ), rapid association and disassociation of KaiA facilitate phosphorylation until the KaiC hexamer is hyperphosphorylated (state $\beta$ ). KaiB is assumed to bind with hyperphosphorylated KaiC; KaiB association with KaiC induces a simultaneous conformational change to a new state $\left(\mathrm{KaiC}^{*}\right.$, state $\chi$ ). The $\mathrm{KaiC}^{*}$ hexamer (state $\chi$ ) dephosphorylates to a relatively hypophosphorylated status (state $\delta$ ) and relaxes to the original conformation (state $\alpha$ ). Simultaneous with the phosphorylation cycle of a hexamer is the possibility of the exchange of monomers between any two hexamers in any of the states. The rates of this exchange may differ depending on the conformational state of the KaiC hexamers, their degree of phosphorylation, and their association with KaiA or KaiB (Fig. 4). We assume that KaiA stochastically binds and unbinds rapidly from KaiC hexamers and that KaiB associates/disassociates with the KaiC hexamer when the total degree of phosphorylation of the hexamer exceeds a threshold that places KaiC in state $\beta$ (for a complete explanation of the model, see Mori et al. 2007).
Within a space of 2 months in early 2007, there were four publications (including ours) that independently derived a similar core KaiC model (Clodong et al. 2007; Mori et al. 2007; van Zon et al. 2007; Yoda et al. 2007). This does not mean that this core model is correct, but it does mean that the parameter space has been thoroughly explored and found to provide robust oscillations. An important difference among the models is the mechanism invoked to explain the excellent maintenance of highamplitude phosphorylation rhythms in vitro. This phenomenon is significant because it requires that the phosphorylation status of each individual KaiC hexamer be synchronized with that of other hexamers in the molecular population. If the hexamers are not synchronized, then the KaiC hexamers will independently free-run and will rapidly get out of phase with each other. In this case, each hexamer may be rhythmic, but the population of hexamers will become asynchronous. Therefore, a mechanism of maintaining synchrony is necessary to explain the sustained in vitro rhythms. In our model (Mori et al. 2007) and that of Yoda and coworkers (2007), phase-dependent exchange of KaiC monomers provides a potential mechanism for synchronizing the number of phosphates on different hexamers of a given conformation. Therefore, oscillation in the phosphorylation of the population of hexamers proceeds in a regulated fashion. The models of Clodong et al. (2007) and van Zon et al. (2007) explain KaiC population synchrony by proposing differential

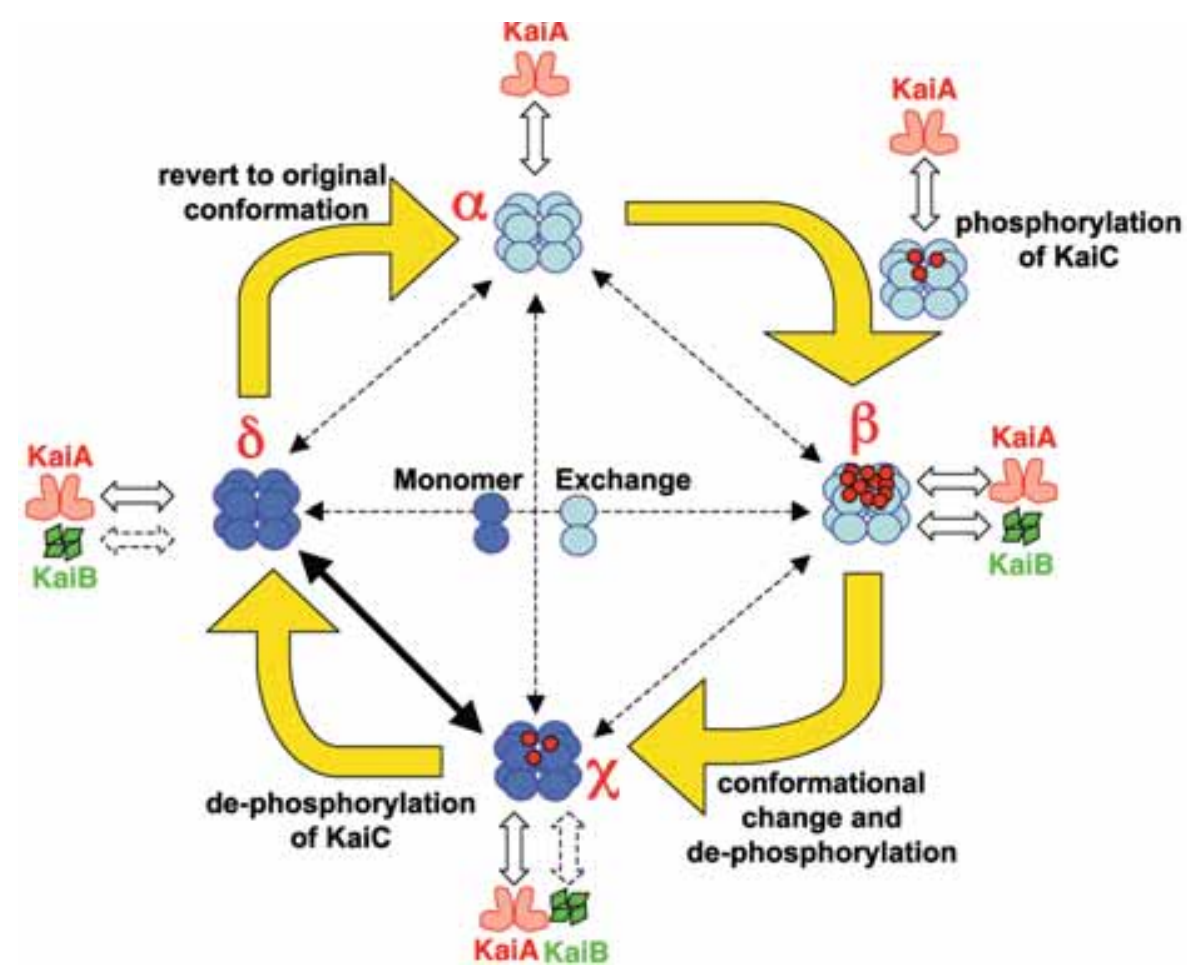

Figure 4. A model for the KaiABC in vitro oscillator. The diagram represents a mathematical model for the phosphorylation cycle of a $\mathrm{KaiC}$ hexamer and its association with KaiA and KaiB. A KaiC monomer is shown as a double circle that can form a hexamer. KaiC hexamers can associate/dissociate with KaiA and/or KaiB. KaiC hexamers are depicted in two conformational states: a default KaiC status (light blue) and an altered $\mathrm{KaiC}^{*}$ state that has undergone a conformational change (darker blue). (Red dots) Phosphates attached to phosphorylation sites on KaiC. KaiC hexamers can exchange monomers between hexamers in any of the states, depicted by the doubleheaded arrows in the central region of the figure. The rate of monomer exchange is maximum during the KaiC dephosphorylation phase (solid double-headed arrow). (Modified, with permission, from Mori et al. 2007 [PLoS Biology].) 
interaction between KaiA and $\mathrm{KaiC}$ that depends on the phosphorylation status of each individual KaiC hexamer. We favor the monomer exchange explanation because it relies upon an experimentally observed phenomenon, whereas the KaiA-KaiC explanation is hypothetical, but these two models of KaiC synchronization are not mutually exclusive and both may be operating.

\section{THE NEXT CHAPTER IN THE BOOK OF BACTERIAL CLOCK STUDIES}

The future is always difficult to predict, but my opinion is that further study of cyanobacterial clock systems will continue to contribute in two major areas: mechanism and evolution/ecology. In the realm of evolution, many unanswered questions remain. The experiments using competition between different strains of $S$. elongatus clearly demonstrate the adaptive significance of having a circadian system (Ouyang et al. 1998; Woelfle et al. 2004), but the mechanistic basis of this strong fitness advantage remains murky. Clarification of the mechanism of this selection could identify the key selective forces involved in the early evolution of circadian clocks, especially because cyanobacteria are thought to be one of the earliest life forms to have evolved on earth (Johnson and Kyriacou 2005). The kai genes that control circadian rhythmicity in S. elongatus are widely distributed among cyanobacterial species (Lorne et al. 2000). Indeed, kaiB and $\mathrm{kaiC}$ homologs are found widely among other eubacteria and even in the archaea (kaiA is less common and is therefore thought to be of more recent evolutionary origin; Dvornyk and Knudsen 2005). Do circadian clocks based on a Kai-type oscillator exist in other types of bacteria? It would be surprising if circadian oscillators do not exist in other bacteria. Cyanobacteria are absolutely dependent on the light/dark cycle for their energy (photosynthesis) and therefore it makes sense that a circadian system was a crucial adaptation; nevertheless, the daily cycle of light (including UV) and temperature must be of critical importance to other types of bacteria and therefore the occurence of kai homologs among other prokaryotes is probably a clue to the existence of bacterial clocks. In the case of Rhodobacter, there is already evidence for a circadian-like rhythm, but temperature compensation has not yet been demonstrated (Min et al. 2005).

Is the kai-based oscillator a prokaryotic phenomenon without evolutionary links to the circadian systems in eukaryotes? When we first started our research on the cyanobacterial clock, we hoped that it would be a "Rosetta Stone" for plant clocks based on the assumption that an ancestral clock from a cyanobacterium might have been transferred or conserved during the endosymbiotic events of plant evolution (Johnson 1994). Unfortunately, that hope was frustrated - we have not found kai homologs in chloroplast or nuclear genomes of plants (there does not even appear to be conservation of clock genes between eukaryotic algae such as Chlamydomonas and eukaryotic plants! [Mittag et al. 2005]). Nevertheless, it remains possible that there is an evolutionary link between prokaryotic and eukaryotic clocks but that tremendous divergence in the sequences of the relevant clock genes has obscured these links so that the links are undetectable by compar- isons within the current repertoire of known prokaryotic and eukaryotic genomes. Perhaps fundamental aspects of clock biochemistry have been conserved between prokaryotes and eukaryotes? It is an intriguing thought.

In the ecological dimension, not only did cyanobacteria transform the atmosphere of Earth from a reducing to an oxidizing environment, but cyanobacteria continue to be a major (probably predominant) contributor to global photosynthesis. Undoubtedly, knowing more about how cyanobacteria accomplish and regulate photosynthesis is important, especially in the face of the impending global warming threat.

In the realm of clock mechanism, the convergence of structural, biophysical, and biochemical studies enabled by the combination of an in vitro system and structural information on key clock proteins will certainly give us the clearest picture in the near future of how "the blind watchmaker" (aka evolution) constructed a circadian clockwork. These insights will be at the most basic molecular level, including visualizing circadian conformational changes of single molecules. In addition to the elucidation of the in vitro oscillator, we need to define the role of a TTFL in cyanobacteria. It is likely that the rhythms of KaiB and KaiC protein abundance (Xu et al. 2003) can feed into the posttranslational oscillator to promote robustness, and the elucidation of the relative contributions of the posttranslational KaiABC oscillator versus the TTFL circuit is of key importance for future studies. The implications of these connections extend beyond the cyanobacterial system. If it is true that the bacterial posttranslational oscillator is embedded within a TTFL and is the underlying determinant of the properties of this circadian system, this organization could also be true for eukaryotic systems. One consequence of the cyanobacterial studies might therefore be to spark a major reevaluation of the evidence supporting a TTFL in eukaryotes-perhaps a totally proteindriven clockwork underlies circadian rhythmicity in eukaryotes (including humans) as well.

Finally, the question of mechanism within the larger clock system of cyanobacteria implicates inputs and outputs. For input, light/dark signals (especially pulses of darkness) and temperature changes can entrain the cyanobacterial clock system. Recent evidence suggests that environment-stimulated alterations of the intracellular redox status may be involved in these input pathways (Ivleva et al. 2006). A particular fascination of the cyanobacterial clock system is how the output rhythm of global gene expression is regulated. As mentioned above, these expression patterns may be mediated by daily changes in the topology of the chromosome such that the activity of all promoters are rhythmically modulated by torsion-sensitive transcription (Mori and Johnson 2001a; Johnson 2004; Smith and Williams 2006; Woelfle and Johnson 2006; Woelfle et al. 2007). In contrast to the cyanobacterial clock, eukaryotic clock control of gene expression is usually described in terms of regulation by the rhythmic activity of specific transcription factors. However, recent evidence indicates that trans- criptional factors that regulate the mammalian clock have an essential histone acetyltransferase activity (Etchegaray et al. 2003; Doi et al. 2006), suggesting that regulation of chromatin structure is an important mechanism for clock con- 
trol of transcription in eukaryotes. Thus, regulation of chromosome structure may be a conserved feature of circadian clocks in general and not a curiosity of the cyanobacterial circadian system.

\section{CONCLUSIONS}

The studies on the cyanobacterial clock system have been pioneering circadian research and continue to be so. First, we now know that eukaryotic organization is not a requirement for the evolution of a circadian system. Cyanobacterial studies provided the first rigorous evidence for the adaptive fitness of circadian programs. We know from cyanobacterial investigations that a strictly posttranslational network of protein interactions is sufficient to confer circadian periodicity and temperature compensation. However, this posttranslational clockwork operates within a larger TTFL network, and the elucidation of the interplay between the posttranslational oscillator and the TTFL may enlighten us regarding eukaryotic clock mechanisms. Cyanobacterial clock proteins are the only ones that have been successfully crystallized and are therefore the only circadian timekeeping proteins to expose their structural secrets. The combination of an in vitro system and structural information has led to a true watershed in the analyses of circadian mechanism, foretelling biophysical and biochemical approaches that will bring circadian analyses of a circadian clockwork to an unprecedented level of molecular detail. Finally, scrutinizing the intriguing orchestration of gene expression by the cyanobacterial clock is likely to lead to new insights into basic mechanisms of gene expression. Are cyanobacterial circadian clocks a mere curiosity? No longer!

\section{ACKNOWLEDGMENTS}

Many scientists contributed to the story described here. Dr. Tsung-Hsien Chen inspired me to study cyanobacterial clocks, which Dr. Takao Kondo and I initiated during Dr. Kondo's sabbatical in my laboratory with the help of Dr. Susan Golden, and later Dr. Masahiro Ishiura. The labs of these four scientists (Kondo, Golden, Ishiura, and Johnson) are largely responsible for establishing the $S$. elongatus system. The cyanobacterial work in my laboratory was performed by Drs. Yao Xu, Tetsuya Mori, Yi Liu, Yan Ouyang, Mark Woelfle, Vladimir Podust, and Ximing Qin with the help of our collaborators Drs. Takao Kondo, Susan Golden, Masahiro Ishiura, Martin Egli, Phoebe Stewart, Hassane Mchaourab, David Piston, Michael Cox, Ross Inman, Rekha Pattanayek, Dewight Williams, and Mark Byrne. Dr. David McCauley has cheerfully consulted on statistical analyses and Dr. Terry Page has been a reliable "sounding board" for discussing circadiana over the years. Finally, I thank my mentors for being scientific muses and role models: J.W. Hastings, Michael Menaker, and Colin Pittendrigh (all of whom were participants at the 1960 Cold Spring Harbor Symposium).

\section{REFERENCES}

Andersson C.A., Tsinoremas N.F., Shelton J., Lebedeva N.V., Yarrow J., Min H., and Golden, S.S. 2000. Application of bio- luminescence to the study of circadian rhythms in cyanobacteria. Methods Enzymol. 305: 527.

Aoki S., Kondo T., Wada H., and Ishiura M. 1997. Circadian rhythm of the cyanobacterium Synechocystis sp. PCC 6803 in the dark. J. Bacteriol. 179: 5751.

Bruce V.G. 1961. Environmental entrainment of circadian rhythms. Cold Spring Harbor Symp. Quant. Biol. 25: 29.

. 1970. The biological clock in Chlamydomonas reinhardtii. J. Protozool. 17: 328.

Chen T.-H., Chen T.-L., Hung L.-M., and Huang T.-C. 1991. Circadian rhythm in amino acid uptake by Synechococcus RF-1. Plant Physiol. 97: 55.

Clodong S., Duhring U., Kronk L., Wilde A., Axmann I., Herzel H., and Kollmann M. 2007. Functioning and robustness of a bacterial circadian clock. Mol. Syst. Biol. 3: 90.

Dodd A.N., Salathia N., Hall A., Kevei E., Toth R., Nagy F., Hibberd J.M., Millar A.J., and Webb A.A. 2005. Plant circadian clocks increase photosynthesis, growth, survival, and competitive advantage. Science 309: 630.

Doi M., Hirayama J., and Sassone-Corsi P. 2006. Circadian regulator CLOCK is a histone acetyltransferase. Cell 125: 497.

Dunlap J.C. 1999. Molecular bases for circadian clocks. Cell 96: 271.

Dvornyk V. and Knudsen B. 2005. Functional divergence of the circadian clock proteins in prokaryotes. Genetica 124: 247.

Edmunds L.N. 1988. Cellular and molecular bases of biological clocks. Springer-Verlag, New York.

Ehret C.F. 1961. Action spectra and nucleic acid metabolism in circadian rhythms at the cellular level. Cold Spring Harbor Symp. Quant. Biol. 25: 149.

Ehret C.F. and Trucco E. 1967. Molecular models for the circadian clock. I. The chronon concept. J. Theor. Biol. 15: 240.

Etchegaray J.P., Lee C., Wade P.A., and Reppert S.M. 2003. Rhythmic histone acetylation underlies transcription in the mammalian circadian clock. Nature 421: 177.

Garces R.G., Wu N., Gillon W., and Pai E.F. 2004. Anabaena circadian clock proteins KaiA and KaiB reveal potential common binding site to their partner KaiC. EMBO J. 23: 1688.

Grobbelaar N., Huang T.-C., Lin H.Y., and Chow T.J. 1986. Dinitrogen-fixing endogenous rhythm in Synechococcus RF1. FEMS Microbiol. Lett. 37: 173.

Halberg F. and Conner R.L. 1961. Circadian organization and microbiology: Variance spectra and a periodogram on behavior of Escherichia coli growing in fluid culture. Proc. Minn. Acad. Sci. 29: 227.

Hastings J.W. 1961. Biochemical aspects of rhythms: Phase shifting by chemicals. Cold Spring Harbor Symp. Quant. Biol. 25: 131.

Hitomi K., Oyama T., Han S., Arvai A.S., and Getzoff E.D. 2005. Tetrameric architecture of the circadian clock protein KaiB: A novel interface for intermolecular interactions and its impact on the circadian rhythm. J. Biol. Chem. 280: 19127.

Huang T.-C. and Grobbelaar N. 1995. The circadian clock in the prokaryote Synechococcus RF-1. Microbiology 141: 535.

Hunt T. and Sassone-Corsi P. 2007. Riding tandem: Circadian clocks and the cell cycle. Cell 129: 461.

Ishiura M., Kutsuna S., Aoki S., Iwasaki H., Andersson C.R., Tanabe A., Golden S.S., Johnson C.H., and Kondo T. 1998. Expression of a gene cluster kaiABC as a circadian feedback process in cyanobacteria. Science 281: 1519.

Ivleva N.B., Gao T., LiWang A.C., and Golden S.S. 2006. Quinone sensing by the circadian input kinase of the cyanobacterial circadian clock. Proc. Natl. Acad. Sci. 103: 17468.

Johnson C.H. 1994. Illuminating the clock: Circadian photobiology. Semin. Cell Biol. 5: 355 .

2004. Global orchestration of gene expression by the biological clock of cyanobacteria. Genome Biol. 5: 217.

. 2005. Testing the adaptive value of circadian systems. Methods Enzymol. 393: 818.

Johnson C.H. and Kondo T. 2001. Circadian rhythms in unicellular organisms. In Handbook of behavioral neurobiology, vol. 13: Developmental psychobiology (ed. J.S. Takahashi et al.), Chap. 3, p. 61. Plenum Press, New York.

Johnson C.H. and Kyriacou C.P. 2005. Clock evolution and adaptation: Whence and whither? In Endogenous plant 
rhythms (ed. A.J.W. Hall and H. McWatters), chap. 10, p. 237. Blackwell Publishing, Oxford, United Kingdom.

Johnson C.H., Elliott J.A., and Foster R.G. 2003. Entrainment of circadian programs. Chronobiol. Int. 20: 741.

Johnson C.H., Golden S.S., Ishiura M., and Kondo T. 1996. Circadian clocks in prokaryotes. Mol. Microbiol. 21: 5 .

Kageyama H., Nishiwaki T., Nakajima M., Iwasaki H., Oyama T., and Kondo T. 2006. Cyanobacterial circadian pacemaker: Kai protein complex dynamics in the KaiC phosphorylation cycle in vitro. Mol. Cell 23: 161.

Katayama M., Tsinoremas N.F., Kondo T., and Golden S.S. 1999. сртA, a gene involved in an output pathway of the cyanobacterial circadian system. J. Bacteriol. 181: 3516.

Kippert F. 1986. Endocytobiotic coordination, intracellular calcium signalling, and the origin of endogenous rhythms. Ann. N.Y. Acad. Sci. 503: 476

Kondo T. and Ishiura M. 1994. Circadian rhythms of cyanobacteria: Monitoring the biological clocks of individual colonies by bioluminescence. J. Bacteriol. 176: 1881-1885.

Kondo T., Mori T., Lebedeva N.V., Aoki S., Ishiura M., and Golden S.S. 1997. Circadian rhythms in rapidly dividing cyanobacteria. Science 275: 224.

Kondo T., Tsinoremas N.F., Golden S.S., Johnson C.H., Kutsuna S., and Ishiura M. 1994. Circadian clock mutants of cyanobacteria. Science 266: 1233.

Kondo T., Strayer C.A., Kulkarni R.D., Taylor W., Ishiura M., Golden S.S., and Johnson C.H. 1993. Circadian rhythms in prokaryotes: Luciferase as a reporter of circadian gene expression in cyanobacteria. Proc. Natl. Acad. Sci. 90: 5672.

Liu Y., Tsinoremas N.F., Golden S.S., Kondo T., and Johnson C.H. 1996. Circadian expression of genes involved in the purine biosynthetic pathway of the cyanobacterium Synechococcus sp. strain PCC 7942. Mol. Microbiol. 20: 1071.

Liu Y., Tsinoremas N.F., Johnson C.H., Lebedeva N.V., Golden S.S., Ishiura M., and Kondo T. 1995. Circadian orchestration of gene expression in cyanobacteria. Genes Dev. 9: 1469.

Lorne J., Scheffer J., Lee A., Painter M., and Miao V.P. 2000. Genes controlling circadian rhythm are widely distributed in cyanobacteria. FEMS Microbiol. Lett. 189: 129.

Min H., Guo H., and Xiong J. 2005. Rhythmic gene expression in a purple photosynthetic bacterium, Rhodobacter sphaeroides. FEBS Lett. 579: 808.

Mitsui A., Kumazawa S., Takahashi A., Ikemoto H., and Arai T. 1986. Strategy by which nitrogen-fixing unicellular cyanobacteria grow photoautotrophically. Nature 323: 720.

Mittag M., Kiaulehn S., and Johnson C.H. 2005. The circadian clock in Chlamydomonas reinhardtii: What is it for? What is it similar to? Plant Physiol. 137: 399.

Mori T. and Johnson C.H. 2000. Circadian control of cell division in unicellular organisms. Prog. Cell Cycle Res. 4: 185.

2001a. Circadian programming in cyanobacteria. Semin. Cell Dev. Biol. 12: 271.

$2001 \mathrm{~b}$. Independence of circadian timing from cell division in cyanobacteria. J. Bacteriol. 183: 2439.

Mori T., Binder B., and Johnson C.H. 1996. Circadian gating of cell division in cyanobacteria growing with average doubling times of less than 24 hours. Proc. Natl. Acad. Sci. 93: 10183.

Mori T., Williams D.R., Byrne M.O., Qin X., Mchaourab H.S., Egli M., Stewart P.L., and Johnson C.H. 2007. Elucidating the ticking of an in vitro circadian clockwork. PLoS Biol. 5: e93.

Nakahira Y., Katayama M., Miyashita H, Kutsuna S., Iwasaki H., Oyama T., and Kondo T. 2004. Global gene repression by KaiC as a master process of prokaryotic circadian system. Proc. Natl. Acad. Sci. 101: 881.

Nakajima M., Imai K., Ito H., Nishiwaki T., Murayama Y., Iwasaki H., Oyama T., and Kondo T. 2005. Reconstitution of circadian oscillation of cyanobacterial KaiC phosphorylation in vitro. Science 308: 414.

Nishiwaki T., Satomi Y., Nakajima M., Lee C., Kiyohara R., Kageyama H., Kitayama Y., Temamoto M., Yamaguchi A., Hijikata A., Go M., Iwasaki H., Takao T., and Kondo T. 2004. Role of KaiC phosphorylation in the circadian clock system of Synechococcus elongatus PCC 7942. Proc. Natl. Acad. Sci. 101: 13927.
Ouyang Y., Andersson C.R., Kondo T., Golden S.S., and Johnson C.H. 1998. Resonating circadian clocks enhance fitness in cyanobacteria. Proc. Natl. Acad. Sci. 95: 8660.

Pattanayek R., Wang J., Mori T., Xu Y., Johnson C.H., and Egli M. 2004. Visualizing a circadian clock protein: Crystal structure of KaiC and functional insights. Mol. Cell 15: 375.

Pattanayek R., Williams D.R., Pattanayek S., Xu Y., Mori T. Johnson C.H., Stewart P.L., and Egli M. 2006. Analysis of KaiA-KaiC protein interactions in the cyano-bacterial circadian clock using hybrid structural methods. EMBO J. 25: 2017.

Pittendrigh C.S., Bruce V.G., Rosensweig N.S., and Rubin M.L. 1959. Growth patterns in Neurospora: A biological clock in Neurospora. Nature 184: 169.

Schweiger H.-G. and Schweiger M. 1977. Circadian rhythms in unicellular organisms: An endeavor to explain the molecular mechanism. Int. Rev. Cytol. 51: 315.

Smith R.M. and Williams S.B. 2006. Circadian rhythms in gene transcription imparted by chromosome compaction in the cyanobacterium Synechococcus elongatus. Proc. Natl. Acad. Sci. 103: 8564.

Stal L.J. and Krumbein W.E. 1985. Nitrogenase activity in the non-heterocystous cyanobacterium Oscillatoria sp. grown under alternating light-dark cycles. Arch. Microbiol. 143: 67.

Sturtevant R.P. 1973. Circadian variability in Klebsiella demonstrated by cosinor analysis. Int. J. Chronobiol. 1: 141.

Sweeney B.M. 1961. The photosynthetic rhythm in single cells of Gonyaulax polyedra. Cold Spring Harbor Symp. Quant. Biol. 25: 145.

Sweeney B.M. and Borgese M.B. 1989. A circadian rhythm in cell division in a prokaryote, the cyanobacterium Synechococcus WH7803. J. Phycol. 25: 183.

Sweeney B.M. and Hastings J.W. 1961. Effects of temperature upon diurnal rhythms. Cold Spring Harbor Symp. Quant. Biol. 25: 87.

Taylor W.R. 1979. "Studies on the bioluminescent glow rhythm of Gonyaulax polyedra." Ph.D. thesis, University of Michigan, Ann Arbor.

Tomita J., Nakajima M., Kondo T., and Iwasaki H. 2005. Circadian rhythm of KaiC phosphorylation without transcription-translation feedback. Science 307: 251.

Vakonakis I. and LiWang A.C. 2004. Structure of the C-terminal domain of the clock protein KaiA in complex with a KaiCderived peptide: Implications for KaiC regulation. Proc. Natl. Acad. Sci. 101: 10925 .

Vakonakis I., Sun J., Wu T., Holzenburg A., Golden S.S., and LiWang A.C. 2004. NMR structure of the KaiC-interacting Cterminal domain of KaiA, a circadian clock protein: Implications for the KaiA-KaiC Interaction. Proc. Natl. Acad. Sci. 101: 1479

van Zon J.S., Lubensky D.K., Altena P.R., and ten Wolde P.R. 2007. An allosteric model of circadian KaiC phosphorylation. Proc. Natl. Acad. Sci. 104: 7420.

Woelfle M.A. and Johnson C.H. 2006. No promoter left behind: Global orchestration of circadian gene expression in cyanobacteria. J. Biol. Rhythms 21: 419.

Woelfle M.A., Ouyang Y., Phanvijhitsiri K., and Johnson C.H. 2004. The adaptive value of circadian clocks: An experimental assessment in cyanobacteria. Curr. Biol. 14: 1481.

Woelfle M.A., Xu Y., Qin X., and Johnson C.H. 2007. Circadian rhythms of superhelical status of DNA in cyanobacteria. Proc. Natl. Acad. Sci. (in press).

Xu Y., Mori T., and Johnson C.H. 2003. Cyanobacterial circadian clockwork: Roles of KaiA, KaiB, and the kaiBC promoter in regulating KaiC. EMBO J. 22: 2117.

$\mathrm{Xu}$ Y., Mori T., Pattanayek R., Pattanayek S., Egli M., and Johnson C.H. 2004. Identification of key phosphorylation sites in the circadian clock protein KaiC by crystallographic and mutagenetic analyses. Proc. Natl. Acad. Sci. 101: 13933.

Ye S., Vakonakis I., Ioerger T.R., LiWang A.C., and Sacchettini J.C. 2004. Crystal structure of circadian clock protein KaiA from Synechococcus elongatus. J. Biol. Chem. 279: 20511.

Yoda M., Eguchi K., Terada T.P., and Sasai M. 2007. Monomershuffling and allosteric transition in KaiC circadian oscillation. PLOS ONE 2: e408. 


\section{$8_{\mathrm{CSH}}^{\infty} \mathrm{C}$ Cold Spring Harbor Symposia SYMPOSIA}

\section{Bacterial Circadian Programs}

C. H. Johnson

Cold Spring Harb Symp Quant Biol 2007 72: 395-404

Access the most recent version at doi:10.1101/sqb.2007.72.027

References This article cites 70 articles, 29 of which can be accessed free at: http://symposium.cshlp.org/content/72/395.full.html\#ref-list-1

\section{License}

Email Alerting

Receive free email alerts when new articles cite this article - sign up in the box at the Service top right corner of the article or click here.

To subscribe to Cold Spring Harbor Symposia on Quantitative Biology go to: http://symposium.cshlp.org/subscriptions 